a custom Visual Basic for Applications (VBA) tools. Intersection and address geocoding was performed using ArcGIS, StreetMap Pro 2003 digital street network, and Google Earth Pro. VBA was used to incorporate offset direction and distance. The geocoding match rate was $99.8 \%$ for postmile-coded collisions and $86 \%$ for intersection-coded collisions (91\% overall).

Conclusions Our framework for geocoding collision data can be applied to data from a variety of settings. Geocoded collision data allow better visualisation of collision occurrence and can facilitate studies of environmental, land use and social characteristics, for which data must be geographically linked.

\section{A FRAMEWORK FOR GEOCODING TRAFFIC COLLISION} DATA

J M Bigham* T M Rice Correspondence: University of California, Berkeley, Safe Transportation Research \& Education Center, 2614 Dwight Way \#7374 Berkeley, CA, USA

10.1136/ip.2010.029215.462

Background Collision geocoding is the process of assigning latitude and longitude coordinates to a traffic collision record. In California and most other US states, collision location is recorded on police reports relative to highway postmile markers or street intersections. In other developed counties, collision location is recorded using intersection, postmile, postal address, or parcel lot. In a recently completed study we geocoded police-reported, fatal and severe injury collisions in California for years 1997-2006.

Objective In this presentation we present an overview of our geocoding processes and a geocoding framework that can be used in other jurisdictions.

Results We identified 142007 collisions in a California-wide collision database. Geocoding was completed using several discrete steps. Perl, a general-purpose programming language, was used to standardise street name information. A highway network with postmile values was created in a semiautomated process using 